\title{
Advantages and Challenges in Drug Re-Profiling
}

\section{Prashansa Agrawal ${ }^{*}$}

Department of Chemistry, Case Western Reserve University, Cleveland, Ohio, 44106, USA

*Corresponding author: Prashansa Agrawal, Department of Chemistry, Case Western Reserve University, Cleveland, Ohio, 44106, USA, Tel: +1 2163682404; E-mail: prashansa.agrawal@case.edu

Received date: November 02, 2015; Accepted date: November 03, 2015; Published date: November 09, 2015

Copyright: $\odot 2015$ Agrawal P. This is an open-access article distributed under the terms of the Creative Commons Attribution License, which permits unrestricted use, distribution, and reproduction in any medium, provided the original author and source are credited.

\section{Editorial}

The drug re-profiling, also coined the term repurposing, repositioning, reusing and rediscovery, is the usage of known drugs for new diseases. The main objective of drug re-profiling is to discover methods for using approved drugs or discarded clinical candidates in the treatment of new diseases. Few examples of repositioned drugs include, Celgene's Thalomid ${ }^{\circ}$, which is repositioned thalidomide, and its derivative Revlimid ${ }^{\circ}$ (lenalidomide), are relaunched to the market and signify a combined global revenue stream of more than $\$ 2.8$ billion for Celgene [1]. Since 2007, 30-40\% of drugs or biologics that are introduced to the market in United States were either drugs reprofiled for new indications, reformulations or new amalgamations of existing drugs [2]. Besides this, drug re-profiling has also been performed on a regular basis by some companies such as, Pfizer, Novartis, Eli Lilly, Ore Pharmaceuticals, Biovista, Numedicus, Melior Discovery and SOM Biotech [3].

Drug re-profiling has extra advantage over conventional drug development as it reduces the development cost for the drugs, because they have already been gone through toxicity and other tests such as clinical trials. According to a recent report [4] which is based on a survey of 30 pharmaceutical and biotechnology companies, the cost to re-introduce a repurposed drug averages $\$ 8.4$ million, whereas to relaunch a new formulation of an existing drug in its original indication costs an average $\$ 41.3$ million. They have higher success rate than the original drugs, because of the availability of comprehensive information on their pharmacology, formulation, potential toxicity, safety and adverse drug reaction issues, thereby waning their attrition rate. Since repurposing is based upon previous research and development efforts, new drug candidate could be set for clinical trials quickly, speeding their review process by the Food and Drug Administration (FDA) and if approved, their incorporation into health care and thus diminishing their entire processing time.

In addition, re-profiled drugs can circumvent initial cost and time needed to introduce a drug to the market. As developing a brand-new drug takes massive time, money and effort. Overall, translation of a promising drug candidate into an approved drug often takes more than 15 years. Therefore, it is critical to improve strategies for drug repurposing in order to decrease the time and cost of drugs along with enhancement in their success rates. Furthermore, the rates for repurposed compounds that reach to the market are 25\% from Phase II and $65 \%$ from Phase III clinical trials, in comparison to new molecular entities are $10 \%$ and $50 \%$, respectively.

Drug re-profiling is basically new use of old drugs [5]. It is sustained by two scientific concepts, (a) known target with new medication and (b) known drug with new target. FDA has approved many repurposed drugs [6] and is introduced to the market. Some of the examples include thalidomide by Celgene Corp., Summit, N.J., which was prescribed for nausea and insomnia in pregnant women in 1950, but later approved by FDA for the treatment of leprosy in 1998 as Thalomid. Since its release, Thalomid has become the star product for Celgene. Another one is Merck's finesteride, originally prescribed to treat prostate enlargement, but in 1998, FDA approved it for the cure of hair loss under the brand name Propecia. Chlorpromazine, originally marketed by Rhone Poulenc as an antihistamine. In 1952, Smith Kline licensed the US rights for chlorpromazine and marketed the drug as a sedative and anti-emetic. More recently, CombinatoRx developed CRx-026, a dual action antitumor drug containing chlorpromazine and a second compound in a proprietary composition. CombinatoRx showed chlorpromazine's role in inhibition of an essential mitotic kinesin, a formerly unknown target for chlorpromazine. Azidothymidine, which is used in the treatment of cancer, is now repurposed as an HIV therapeutic and vice versa.

Since drug repurposing largely refers to reviewing drugs that have already been approved for the cure of one disease or condition and perceive if they are safe and effective for treating other diseases. In this process, drug repositioning has some major challenges that still need to overcome. These challenges include, intellectual property rights adjoining the original drug may be complicated and commercially, it may not be possible to take such a drug to market due to a high risk that may be involved in it, novel methods for identifying repositioning opportunities, regulatory issues/timelines and legal frameworks for drug repurposing, product lifecycle management through new drug indications, re-tasking currently marketed drugs through combination therapy, market potential of drug re-profiling and technical challenges of drug repositioning. To overcome these challenges, new complementary approaches are required that can enable repositioning of old drug candidates [7].

Drug re-profiling is becoming an emerging field in the area of drug development. Using old drugs for new cure can be very significant [8], since only one in 10,000 compounds can make it to market, and less than $20 \%$ of drugs entering Phase II clinical trials succeed. In addition, their development timeline (average 10 years) and cost (at least \$1 billion) is high. It will be very beneficial to find a way to repurpose drugs, which are either in the market already or those failed in the clinical trials as it will be time- and cost-effective. The Institute of Medicine released a 118-page report on drug repurposing, observing that many pharmaceutical companies are retrieving failed compounds and seeing whether these drugs might have other indications, predominantly after an increasing number of earlier abandoned compounds that have been repurposed.

However, successful drug repositioning requires a deep understanding of biological mechanisms-from known overlaps of mechanisms to re-innovation of a new molecule, to find a new mechanism, dosing, route of administration, new target. To reap the benefits, drug re-profiling must be executed through a planned process 
Page 2 of 2

based on targeted positioning of cutting-edge invention. Methods that address and diminish the hypothesis risk will be vital to improve the success rate of re-profiled dugs in the new drug and product development process.

\section{References}

1. http://www.conferenceseries.com/

2. Graul AI, Sorbera L, Pina P, Tell M, Cruces E, et al. (2010) The Year's New Drugs and Biologics - 2009. Drug News Perspect 23: 7-36.

3. Sleigh SH, Barton CL (2010) Repurposing Strategies for Therapeutics. Pharmaceutical Medicine 24: 151-159.
4. Pharmaceutical Product Relaunch: Preserving Market Share through Line Extension and New Market Entry Strategies (2007) Cutting Edge Information.

5. Chong CR, Sullivan DJ (Aug 2007) New uses for old drugs. Nature 448: 645-646.

6. Hughes B (2008) 2007 FDA drug approvals: a year of flux. Nature Reviews Drug Discovery 7: 107-109.

7. Tartaglia LA (2006) Complementary new approaches enable repositioning of failed drug candidates. Expert Opinion on Investigational Drugs 15: 1295-1298.

8. Aronson JK (2007) Old drugs - new uses. British Journal of Clinical Pharmacology 64: 563-565. 